\title{
Binding Mechanism of Neutralizing Nanobodies Targeting SARS- CoV-2 Spike Glycoprotein
}

\author{
Mert Golcuk, Aysima Hacisuleyman, Burak Erman, Ahmet Yildiz, and Mert Gur* \\ Cite This: https://doi.org/10.1021/acs.jcim.1c00695 \\ Read Online
}

ABSTRACT: Severe acute respiratory syndrome coronavirus 2 (SARS-CoV-2) enters human cells upon binding of its spike $(S)$ glycoproteins to ACE2 receptors. Several nanobodies neutralize SARS-CoV-2 infection by binding to the receptor-binding domain (RBD) of the S protein, but how their binding antagonizes S-ACE2 interactions is not well understood. Here, we identified interactions between the RBD and nanobodies H11-H4, H11-D4, and Tyl by performing all-atom molecular dynamics simulations. H11-H4 and H11-D4 can bind to RBD without overlapping with ACE2. H11-H4, and to a lesser extent H11-D4, binding dislocates ACE2 from its binding site due to electrostatic repulsion. In comparison, Ty1 overlaps with ACE2 on RBD and has a similar binding strength to ACE2. Mutations in the Alpha variant of SARS-CoV-2 had a minor effect in RBD binding strengths of ACE2 and nanobodies, but reduced the ability of H11-H4 and H11-D4 to dislocate ACE2 from RBD. In comparison, the Beta variant weakened the RBD binding strengths of H11-H4 and H11-D4, which were less effective to dislocate ACE2 binding. Unexpectedly, mutations in Beta strengthened Tyl binding to RBD, suggesting that this nanobody may be more effective to neutralize the Beta variant of SARS-CoV-2.

\section{INTRODUCTION}

Nanobodies are single-domain antibodies that are used in disease diagnosis and as drug carriers. ${ }^{1}$ Because of their low molecular weight $(12-30 \mathrm{kDa})$ and complexity, they can be mass-produced rapidly at a low cost in bacteria or yeast. ${ }^{2,3}$ Nanobodies have unique structures with loop regions (complementarity determining regions, CDRs) that have exquisite chemical and shape complementarity to their protein targets $^{4}$ (SI Appendix, Figure S1). Recent studies identified several nanobodies, including $\mathrm{H} 11-\mathrm{H} 4,{ }^{5} \mathrm{H} 11-\mathrm{D} 4,{ }^{5}$ Ty1, $\mathrm{Nb} 20,{ }^{7} \mathrm{Nb} 6,{ }^{8}$ and $\mathrm{Sb}_{23}{ }^{9}$ as promising therapeutic agents for neutralizing SARS-CoV-2 infection. These nanobodies specifically bind to the receptor-binding domain (RBD) of the spike (S) glycoprotein ${ }^{5-9}$ and block its interaction with the human angiotensin-converting enzyme 2 (ACE2). ${ }^{10,11}$ While Ty1, $\mathrm{Nb} 20,{ }^{7} \mathrm{Nb}_{6}{ }^{8}$ and $\mathrm{Sb} 23^{9}$ sterically overlap with the ACE2 binding site, ${ }^{6-9} \mathrm{H} 11-\mathrm{H} 4^{5}$ and $\mathrm{H} 11-\mathrm{D} 4^{5}$ prevent ACE2 binding without an overlap 5 (Figure 1A). It remains unclear how these antibodies neutralize S-ACE2 interactions with and without a binding site overlap.

Recently, two SARS-CoV-2 variants Alpha (N501Y mutation at $S$ RBD) $)^{12}$ and Beta (N501Y/E484K/K417N mutations at $\mathrm{S} R B D$ ) were observed at a fast-growing rate across the globe. While it remains unclear whether current treatments are effective against these variants, nanobodies can be rapidly engineered using recombinant methods in order to cope with the mutagenesis of the $S$ protein. These studies would be greatly aided by an understanding of how existing nanobodies neutralize the wild-type (WT) $S$ protein at the molecular level.

To address these challenges, we performed all-atom MD simulations totaling $27.6 \mu \mathrm{s}$ in length using the recently solved structures of the RBD of SARS-CoV-2 S protein in complex with the $\mathrm{N}$-terminal peptidase domain (PD) of human $\mathrm{ACE}^{13}{ }^{13}$, the nanobodies H11-H4, ${ }^{5} \mathrm{H} 11-\mathrm{D} 4,{ }^{5} \mathrm{Ty} 1{ }^{6}$ or both the PD and either H11-H4 or H11-D4. We first simulated binding of these nanobodies to WT, N501Y, and N501Y/ $\mathrm{E} 484 \mathrm{~K} / \mathrm{K} 417 \mathrm{~N}$ mutants of RBD. These simulations revealed additional interactions between RBD and the nanobodies to those observed in structural studies. ${ }^{5,6}$ We also simulated the detachment of the nanobodies from $\mathrm{RBD}$ at low pulling speeds $\left(0.1 \AA \mathrm{ns}^{-1}\right)$. These simulations estimated that the binding strength of the nanobodies to RBD is similar to that of ACE2. We next explored how binding of these nanobodies disrupt $S$ ACE2 interactions. While H11-H4 and H11-D4 do not overlap with the ACE2 binding site, simulations of the nanobodyRBD-PD complex revealed that these nanobodies disrupt SACE2 interactions by electrostatic repulsion when they are bound to RBD side by side with PD of ACE2. Unlike these

Received: June 17, 2021 
A
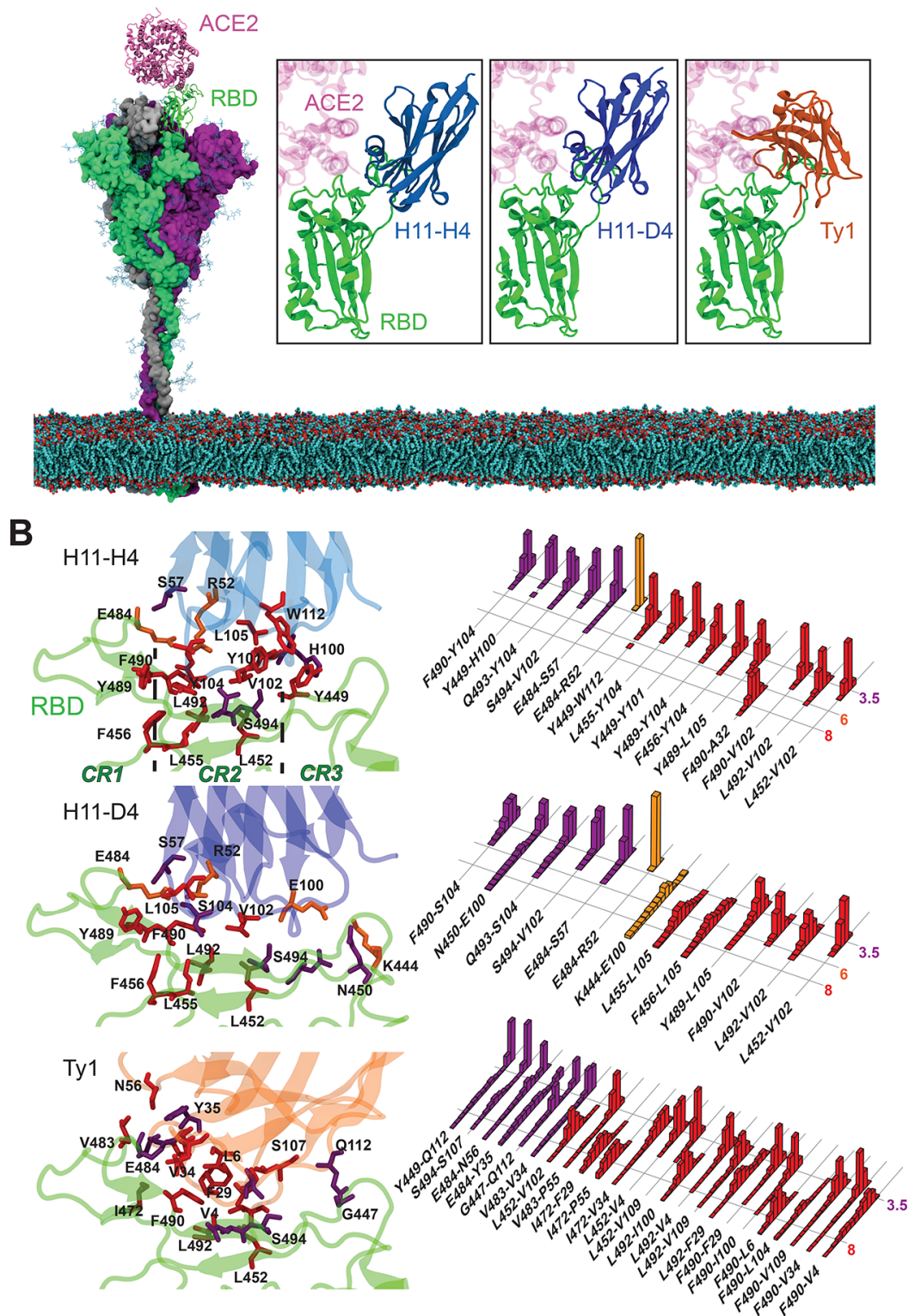

Figure 1. Interactions between RBD of the SARS-CoV-2 S protein and nanobodies. (A) Nanobodies in complex with the RBD of the S protein located on the viral membrane. The atomic models were constructed using the full-length $S$ protein model ${ }^{29}$ and the structure of RBD in complex with ACE2 (PDB 6M0J). ${ }^{13}$ The $S$ protein trimer shown is composed of two protomers with RBD in the down position (gray and purple) and one protomer with RBD in the up position (green). The structures of RBD in complex with the nanobodies H11-H4, H11-D4, and Ty1 (PDB 6ZBP, $6 \mathrm{YZ5}$, and 6ZXN, respectively) ${ }^{5,6}$ are highlighted in boxes. ACE2 is superimposed on RBD based on its coordinates in the crystal structure (PDB $6 \mathrm{MOJ}$ ). Only the RBD of S protein, PD of ACE2, and nanobodies were used in MD simulations. (B) (left) Hydrophobic interactions, hydrogen bonding, and salt bridges between H11-H4, H11-D4, and Ty1, with RBD. (right) Normalized distributions of the distances between the amino-acid pairs that form hydrophobic interactions (red), hydrogen bonds (purple), and salt bridges (orange).

nanobodies, Ty1 disrupts S-ACE2 interactions by overlapping with the ACE2 binding site. Simulations on the N501Y mutant of $S$ protein showed that it has minor effects on RBD-ACE2 and RBD-nanobody interactions. However, the N501Y/ $\mathrm{E} 484 \mathrm{~K} / \mathrm{K} 417 \mathrm{~N}$ triple mutant reduced the binding strength of H11-H4 and H11-D4 while increasing that of Ty1. For both N501Y and N501Y/E484K/K417N mutants, ACE2 was less likely to detach from RBD upon $\mathrm{H} 11-\mathrm{H} 4$ and H11-D4 binding. In particular, ACE2 triggered detachment of $\mathrm{H} 11-\mathrm{H} 4$ from mutant RBDs. These results suggest how existing nanobodies can be reengineered to neutralize RBD mutations that take place during the pandemic.

\section{MATERIALS AND METHODS}

MD Simulation System Preparation. As was done in our previous study, ${ }^{14}$ the structure of SARS-CoV-2 S protein RBD bound with ACE2 at $2.45 \AA$ resolution (PDB ID 6M0J) ${ }^{13}$ was used as a template for the conventional MD (cMD) simulations of the S-ACE2 complex. For the nanobodies, crystal structures (PDB ID 6ZBP), ${ }^{5}$ (PDB ID 6YZ5), ${ }^{5}$ and (PDB ID 6ZXN $)^{6}$ were used as templates for the H11-H4RBD, H11-D4-RBD, and Ty1-RBD systems. We kept the chloride ion, zinc ion, glycans, and water molecules observed in the structures. Regions of glycans that were not visible in structural studies were not included in the simulations. Using 
the TIP3P water model, each system was solvated in a water box with a $25 \AA$ cushion in each direction (50 A water cushion between the protein complexes and their periodic images). For Steered $\mathrm{MD}^{15}$ (SMD) simulations, systems were solvated having $50 \AA$ cushion along the pulling direction in order to create enough space for unbinding simulations and $15 \AA$ cushion in all other directions. Ions were added to neutralize the system and $\mathrm{NaCl}$ concentration was set to $150 \mathrm{mM}$. The size of solvated systems was $\sim 150000$ and $\sim 120000$ atoms for cMD and SMD simulations, respectively. All system preparation steps were performed in VMD. ${ }^{16}$

cMD Simulations. All MD simulations were performed under N, P, T conditions in NAMD $2.14^{17}$ using the CHARMM $36^{18}$ force field with a time step of $2 \mathrm{fs}$. The temperature was kept at $310 \mathrm{~K}$ using Langevin dynamics with a damping coefficient of $1 \mathrm{ps}^{-1}$ while pressure was maintained at 1 atm using the Langevin Nosé-Hoover method with an oscillation period of $100 \mathrm{fs}$ and a damping time scale of $50 \mathrm{fs}$. A $12 \AA$ cutoff distance was used for van der Waals interactions. The particle-mesh Ewald method was used to calculate longrange electrostatic interactions. Furthermore, periodic boundary conditions were applied in all simulations. Each system was first minimized for 10000 steps and subsequently equilibrated for $2 \mathrm{~ns}$ by keeping the protein fixed. A second minimization-equilibration cycle was performed: The complete system was minimized for additional 10000 steps without fixing the protein, which was followed by $4 \mathrm{~ns}$ of equilibration by applying harmonic constraints on $\mathrm{C}_{\alpha}$ atoms. As a final step prior to production runs, these constraints were released, and the system was equilibrated for an additional 4 ns. These simulations are expected to account for the structural differences due to the radically different thermodynamic conditions of crystallization solutions and MD simulations. ${ }^{19}$ MD simulations were performed in supercomputers Comet, Expanse, and Stampede2 using a total of $\sim 10$ million corehours.

Criteria for Interactions. As a salt bridge formation criterion, a cutoff distance of $6 \AA$ between the basic nitrogen and acidic oxygen was used. ${ }^{20} \mathrm{~A}$ maximum $3.5 \AA$ distance between hydrogen bond donor and acceptor and a $30^{\circ}$ angle between the hydrogen atom, the donor heavy atom, and the acceptor heavy atom was used to score a hydrogen bond formation. $^{21}$ Interaction pairs that did not satisfy the angle criterion but did satisfy the distance criterion were classified as electrostatic interactions. A cutoff distance of $8 \AA$ between the side chain carbon atoms was used for hydrophobic interactions. ${ }^{22-24}$

SMD Simulations. SMD simulations were performed at a constant pulling velocity of $0.1 \AA \mathrm{ns}^{-1}$. A dummy atom, which is pulled at a constant velocity along the "pulling" direction, is attached to the center of mass of "steered" atoms by a virtual spring. The resulting force $\mathbf{F}$ applied to the steered atoms depends on the instantaneous (at time $t$ ) center of mass coordinates of steered atoms $\mathbf{R}$ as follows,

$$
\begin{aligned}
& \mathbf{F}=-\nabla U \\
& U=\frac{1}{2} k\left[\mathbf{v} t-\left(\mathbf{R}-\mathbf{R}_{0}\right) \cdot \mathbf{n}\right]^{2}
\end{aligned}
$$

where $U$ is the guiding potential, $k$ is the spring constant, $\mathbf{v}$ is the pulling velocity, $t$ is time, $\mathbf{n}$ is the direction of pulling, and $\mathbf{R}_{0}$ is the coordinate of the center of mass of steered atoms at time $0 .{ }^{25}$ Work performed is evaluated by integrating $\mathbf{F}$ over displacement $\xi$ along the pulling direction. Steered and fixed atoms were selected as the $\mathrm{C}_{\alpha}$ atoms at the nanobody-RBD and $\mathrm{RBD}-\mathrm{ACE} 2$ interface. Please refer to the Supporting Information regarding detailed information for the steered and fixed atoms (SI Appendix Table S2). The vector pointing from the center of mass of fixed atoms to the center of mass of steered atoms was selected as pulling direction. At a spring constant of $100 \mathrm{kcal} \mathrm{mol}^{-1} \AA^{-2}$, the center of mass of the steered atoms followed the dummy atom closely while the spring was still soft enough to allow small deviations (satisfying the stiff spring approximation). ${ }^{26}$ Each SMD simulation was performed for $300 \mathrm{~ns}$ which equals a pulling distance of $30 \AA$. Starting conformations of SMD simulations were taken from 100 and $200 \mathrm{~ns}$ time instances of cMD simulations.

\section{RESULTS AND DISCUSSION}

Interaction Network between the RBD and Nanobodies. To model the dynamic interactions of the nanobodyRBD binding interface, we used the costructures of RBD of the SARS-CoV-2 S protein in complex with H11-H4, ${ }^{5}$ H11-D4, ${ }^{5}$ and $\mathrm{Ty}^{6}$ (Figure 1A). These structures were solvated in a water box that contains a physiologically relevant salt concentration (150 $\mathrm{mM} \mathrm{NaCl})$. For each nanobody-RBD complex, two sets of cMD simulations, each of $200 \mathrm{~ns}$ in length, were performed to determine the formation of salt bridges $^{20}$ and hydrogen bonds, as well as electrostatic and hydrophobic interactions between RBD of S protein and PD of ACE2 (see Materials and Methods). Observation frequencies were classified as high and moderate for interactions that occur in $49 \%$ and above and between 15 and $48 \%$ of the total trajectory, respectively. ${ }^{14}$

Previously, we divided the RBD-ACE2 interaction surface into three contact regions (CR1-3, Figure 1B) and proposed that $\mathrm{RBD}-\mathrm{ACE} 2$ interaction is primarily stabilized by hydrophobic interactions in CR1. ${ }^{14,27}$ Similar to ACE2, we observed that nanobodies form many pairwise interactions with RBD. H11-H4 makes 10 hydrophobic interactions, (Figure 1B), 5 hydrogen bonds (Figure 1B), 1 salt bridge, and 1 electrostatic interaction with RBD (Figure 1B). CR1 of RBD comprised about half of these interactions. In comparison, CR1 comprised 5 out of 14 interactions ( 5 hydrophobic interactions, 5 hydrogen bonds, 2 salt bridges, and 2 electrostatic interactions) between H11-D4 and RBD (Figure 1B). Compared to H11-H4 and H11-D4, Ty1 forms a higher number of interactions (18 hydrophobic interactions, 6 hydrogen bonds, and 8 electrostatic), most of which are located in CR1 (Figure 1B).

The interaction network we identified between the nanobodies and RBD is mostly consistent with reported structures. ${ }^{5,6}$ However, we observed an additional three hydrophobic interactions (F490-V102, F490-A32, Y449W112; RBD-nanobody) and 1 hydrogen bond (Q493-Y104) for H11-H4, two hydrophobic interactions (F490-V102, L455-L105), and one salt bridge (K444-E100) for H11D4, and six hydrophobic interactions (L452-V102, L452-V4, L452-V109, L492-F29, L492-V4, L492-I100) and two hydrogen bonds (S494-S107 and G447-Q112) for Ty1. This discrepancy may be due to different thermodynamic conditions between structural studies versus MD simulations, which are performed under physiological conditions. ${ }^{19}$ Alternatively, MD simulations performed with explicit water molecules (TIP3P) may overestimate the stability of the salt bridges relative to experimental studies. $^{28}$ 

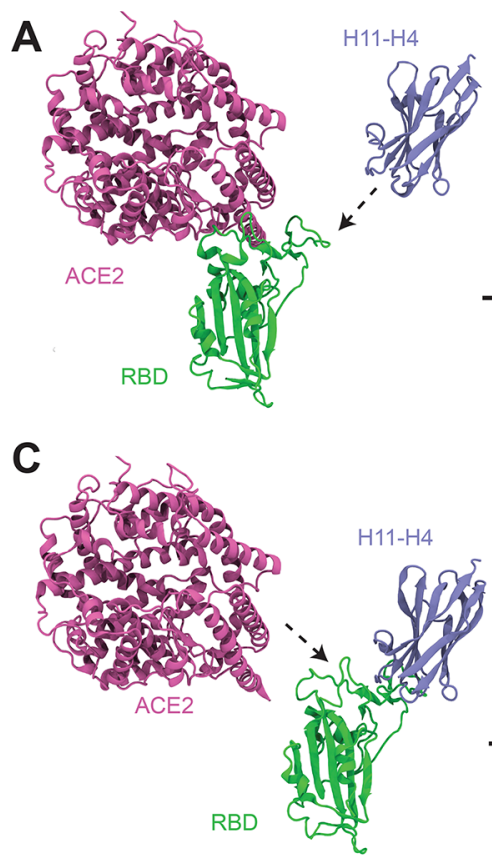

E
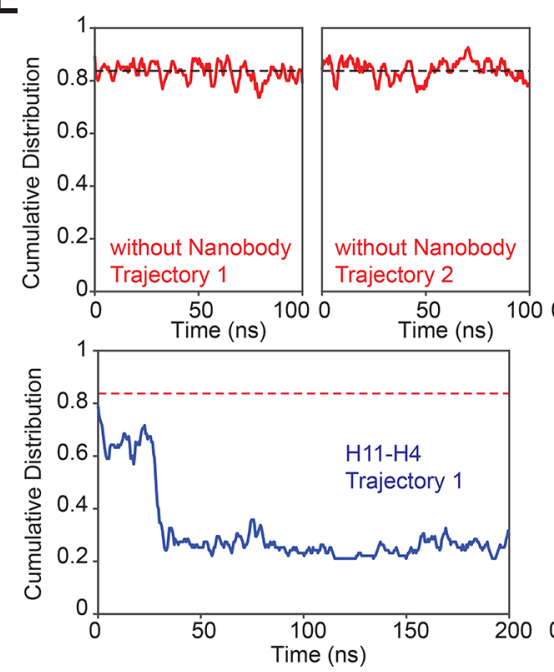
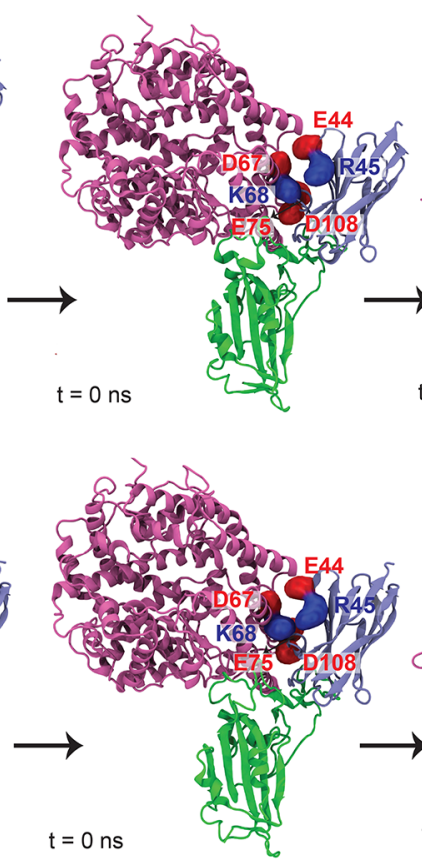

$=200 \mathrm{~ns}$

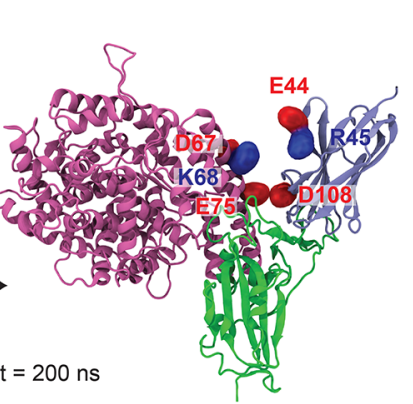

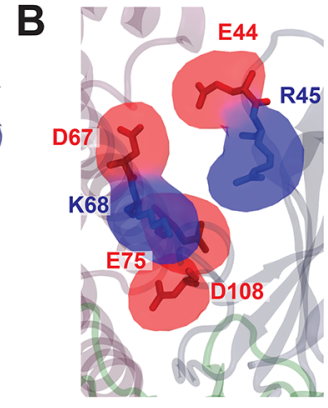

D

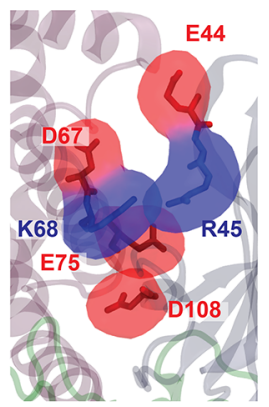

$\mathbf{F}$
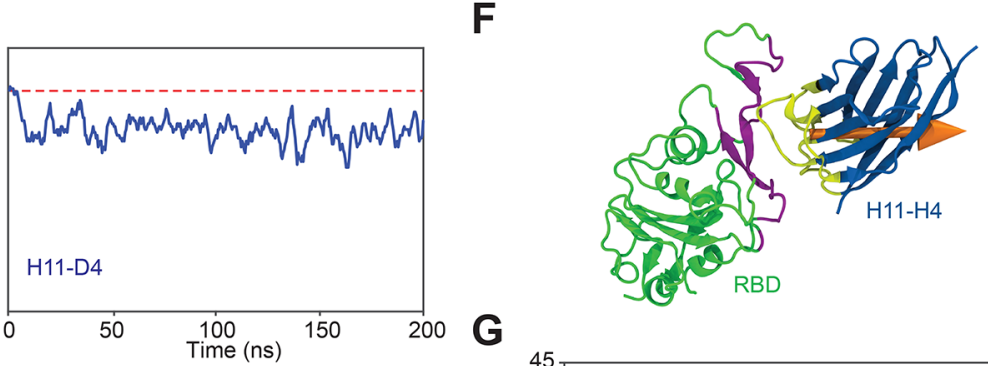

G
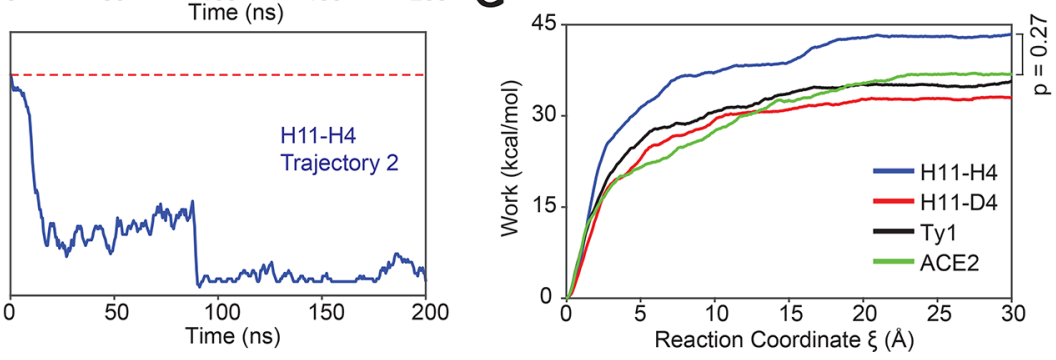

Figure 2. Effect of H11-H4 and H11-D4 binding on interactions between RBD and ACE2. (A) H11-H4 is docked onto the RBD/ACE2 complex. (B) Electrostatic repulsion between ACE2 and H11-H4 upon H11-H4 docking. (C) ACE2 is docked onto the RBD/H11-H4 complex. (D) Electrostatic repulsion between ACE2 and H11-H4 upon ACE2 docking. Neighboring ACE2 and H11-H4 residues with identical charges are highlighted in surface representation in red (negatively charged) and blue (positively charged). Electrostatic repulsion among these charges dislocates ACE2 from its binding site. (E) Pairwise interactions between RBD and ACE2 were normalized by the total numbers of possible interactions. In the absence of the nanobodies, RBD and ACE2 stably maintained $\sim 84 \%$ of these interactions on average (dotted line). The docking of a nanobody to the existing ACE2-RBD complex resulted in a substantial reduction of pairwise interactions between RBD and ACE2 (blue traces). Time zero indicates the time instant after equilibration. (F) In SMD simulations, $\mathrm{C}_{\alpha}$ atoms of RBD residues (purple) were fixed, whereas $\mathrm{C}_{\alpha}$ atoms of $\mathrm{H} 11-\mathrm{H} 4$ (yellow) were steered at a constant pulling velocity (orange arrow) of $0.1 \AA \mathrm{ns}^{-1}$. (G) Average work required to move a nanobody or ACE2 away from RBD along the reaction coordinate. The unbinding work for ACE2 is taken from our previous RBD pulling simulations performed under similar conditions. ${ }^{14}$

ACE2 Releases from RBD upon Nanobody Binding. To understand how targeting RBD with these nanobodies disrupt S-ACE2 interactions, we first superimposed them together with ACE2 on RBD. We observed that H11-H4 and H11-D4 do not overlap with the ACE2 binding site, whereas Ty1 sterically overlaps with ACE2 (Figure 1A). To investigate how H11-H4 and H11-D4 could disrupt S-ACE2 binding without an overlap, we manually docked the structural coordinates of the nanobodies from their costructure with $\mathrm{RBD}^{5}$ onto the RBD-ACE2 complex (Figure $2 \mathrm{~A}$ and $\mathrm{B}$ ). We also altered the order of binding by docking the coordinates of ACE2 (PDB ID 6MOJ) ${ }^{13}$ onto the RBD-nanobody (PDB ID 6ZBP, 6YZ5) ${ }^{5}$ structures (Figure 2C and D). Docking was performed either before (i.e., using the protein structures) or after running cMD simulations for $100 \mathrm{~ns}$ in solution conditions.

For H11-H4, we ran six 200 ns-long cMD simulations under different docking orders. In these simulations, H11-H4 binding to RBD abrogated up to $95 \%$ of the high-frequency interactions between $\mathrm{RBD}$ and $\mathrm{ACE} 2^{14}$ (Figure $2 \mathrm{E}$ and SI 


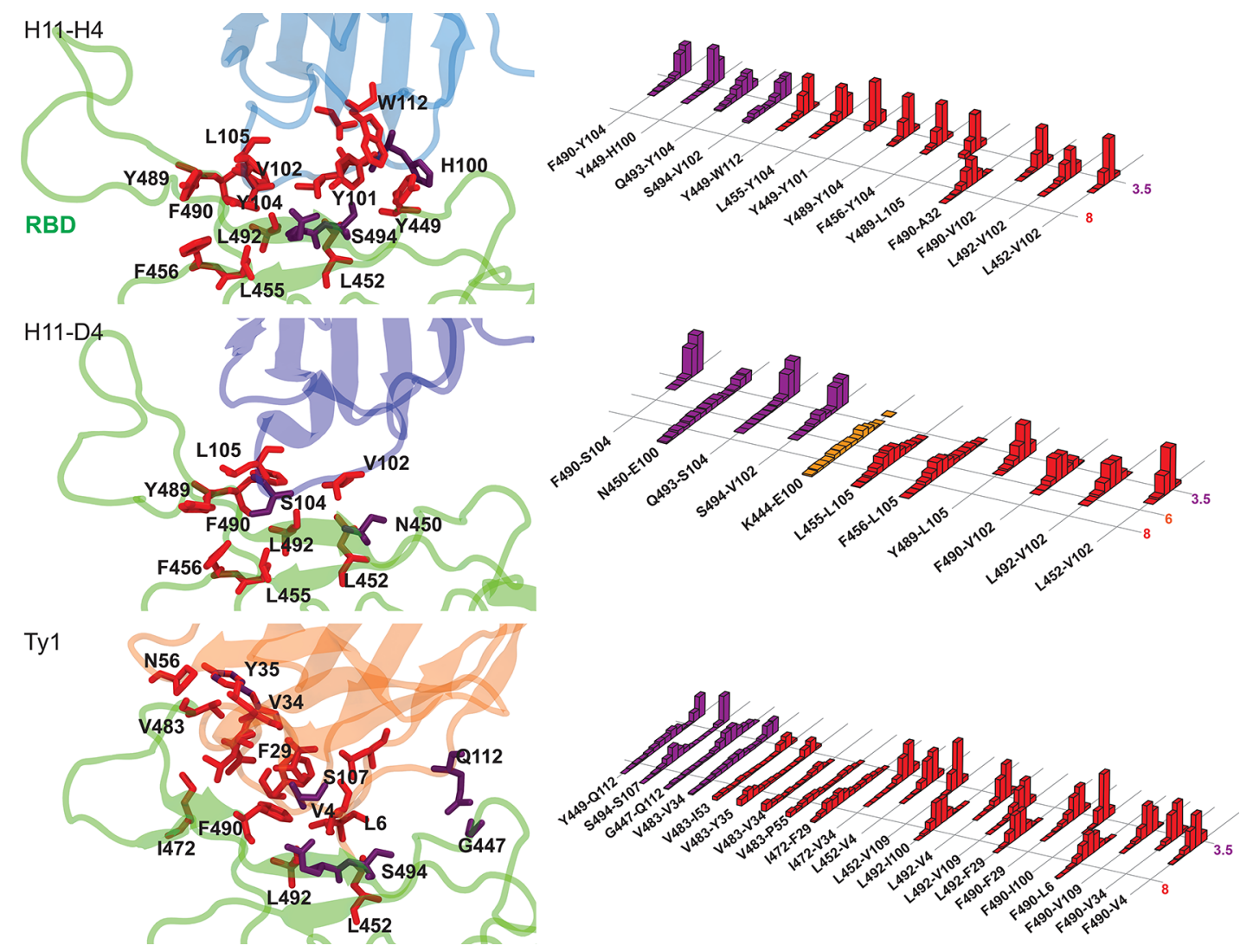

Figure 3. Interactions between the N501Y/E484K/K417N mutant of RBD and the nanobodies. (Left) Hydrophobic interactions, hydrogen bonding, and salt bridges between RBD and H11-H4, H11-D4, or Ty1. (Right) Normalized distributions of the distances between the amino-acid pairs that form hydrophobic interactions (red), hydrogen bonds (purple), and salt bridges (orange).

Appendix Figures S2 and S3). In all cases, ACE2 first detached from CR1, and then lost its binding pose, and was partially unbound from RBD (SI Appendix Movie S1). Close examination of the RBD-ACE2-nanobody conformers indicated no structural changes in RBD (SI Appendix Figure S4) and suggested that nanobody binding does not substantially alter the interaction surface between ACE2 and RBD through allosteric communication. Almost all pairwise interactions between $\mathrm{RBD}$ and ACE2 remain unaltered upon initial binding, except the E484-K31 interaction which was disrupted by the CDR3 loop of H11-H4. We noticed that identically charged residues of ACE2 and nanobodies (D67 with E44, K68 with R45, and E75 with D108; ACE2 with H11$\mathrm{H} 4)$ come in close vicinity and repel each other when both proteins are bound to RBD side by side (Figure $2 \mathrm{~B}$ and $\mathrm{D}$ and SI Appendix Movie S1). In all simulations, H11-H4 won the "tug-of-war" (defined as the reduction of RBD interactions below 45\%) and moved ACE2 away from its binding site.

Similar to H11-H4, docking of H11-D4 on the RBD-ACE2 conformer (PDB ID 6M0J, ${ }^{13} 6 \mathrm{YZ5}^{5}$ ) caused electrostatic repulsion between D67-E44, K68-R45, and E75-D108 (ACE2-H11-D4) residues. However, H11-D4 docking resulted in the disruption of fewer $\mathrm{RBD}-\mathrm{ACE} 2$ interactions and ACE2 remained bound to RBD in most of the 200 ns simulation time of two cMD simulations (Figure $2 \mathrm{E}$ and $\mathrm{SI}$ Appendix Figures S2 and S3). These results suggest that H11$\mathrm{H} 4$ is more effective than H11-D4 in preventing RBD binding to ACE2, consistent with the higher neutralizing activity of H11-H4 against SARS-CoV-2 infection. ${ }^{5}$
Force-Induced Detachment of the Nanobodies from RBD. To estimate the $\mathrm{RBD}$ binding strength of the nanobodies, we performed SMD simulations by pulling nanobodies at constant velocities along the vector pointing away from the binding interface (Figure $2 \mathrm{~F}$ ). In comparison to typical pulling speeds used in MD studies $(2-50 \AA$ $\left.\mathrm{ns}^{-1}\right),{ }^{14,30-38}$ we pulled nanobodies at lower speeds $(0.1 \AA$ $\mathrm{ns}^{-1}$ ) comparable to high-speed atomic force microscopy (AFM) experiments to better estimate the unbinding free energy. ${ }^{14,39}$

For each nanobody, four $300 \mathrm{~ns}$ long SMD simulations (SI Appendix Table S1) were initiated from different conformations sampled from cMD simulations. The average work applied to unbind H11-H4, H11-D4, and Ty1 from RBD was $43.4 \pm 10.7,33.0 \pm 6.4$, and $35.7 \pm 6.3 \mathrm{kcal} / \mathrm{mol}($ mean \pm s.d.), respectively. These values are comparable to $36.8 \pm 9.4$ $\mathrm{kcal} / \mathrm{mol}$ work required to unbind RBD from ACE2 under the same pulling speed ( $p \geq 0.27$, two-tailed $t$ test; Figure $2 \mathrm{G}){ }^{14}$

Nanobody and ACE2 Interactions of the N501Y and N501Y/E484K/K417N Mutant. Next, we performed cMD and SMD simulations to investigate how ACE2 and nanobody binding is affected by N501Y and N501Y/E484K/K417N mutations on RBD. The total number of interactions between the nanobodies and N501Y RBD (SI Appendix Figure S5) is similar to that between the nanobodies and WT RBD (Figure 1B), mainly because N501Y is located on the ACE2-RBD interface and positioned away from the nanobody binding interface. However, H11-H4 and H11-D4 make 3 and 4 fewer interactions, respectively, with the N501Y/E484K/K417N mutant of RBD (Figure 3). In contrast, Ty1 forms 2 additional 

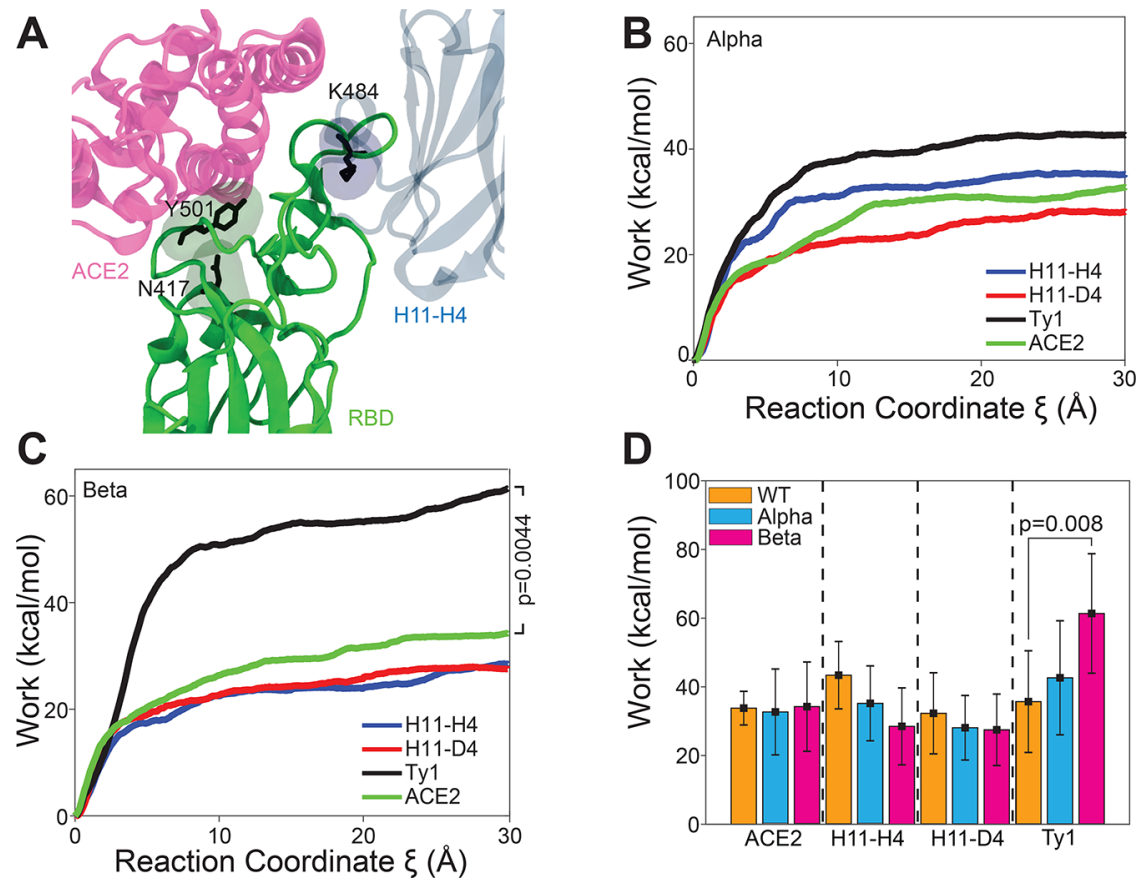

Figure 4. Force-induced detachment of the nanobodies from mutant and WT S proteins. (A) Locations of N501Y, E484K, and K417N mutations. Average work required to unbind nanobodies or ACE2 from the (B) N501Y and (C) N501Y/E484K/K417N mutants. (D) Change of unbinding work values due to RBD mutations. Error bars represent standard deviation.

hydrophobic interactions and 2 fewer hydrogen bonds with the N501Y/E484K/K417N mutant, compared to WT RBD (Figure 3). The changes in number of interactions was primarily due to the $\mathrm{E} 484 \mathrm{~K}$ mutation, which is located on the nanobody-RBD binding interface (Figure 4A, SI Appendix Figure S6A and B).

We ran SMD simulations to investigate how these mutations affect the RBD binding strength of ACE2 and the nanobodies. We found that ACE2 binds to these mutants as strongly as it binds to WT RBD (Figure 4B-D). Although we observed a slight decrease in the binding strength of H11-H4 and H11-D4 (19\% and 15\%, respectively; Figure 4B and D) and a slight increase (19\%) in Ty1 binding strength to N501Y RBD, these changes were not statistically different $(p>0.13$, a two-tailed $t$ test). To test whether the lack of significance is due to low sampling of starting conformations, we also ran 20 SMD simulations at higher pulling speeds $\left(2 \AA \mathrm{ns}^{-1}\right)$ for each nanobody. As expected, an increase in pulling speed resulted in higher unbinding work values (SI Appendix Figure S6D and F). Consistent with SMD simulations performed at $0.1 \AA \mathrm{ns}^{-1}$, simulations at $2 \AA \mathrm{ns}^{-1}$ resulted in a modest decrease in H11$\mathrm{H} 4$ and H11-D4 binding strength and a modest increase in Ty1 binding strength, but these differences were also not statistically significant $(p>0.05$, two-tailed $t$ test $)$. We concluded that the N501Y mutation did not substantially alter the RBD binding strengths of the nanobodies and ACE2.

Unlike N501Y RBD, the N501Y/E484K/K417N triple mutations of RBD decreased the binding strengths of H11$\mathrm{H} 4$ and H11-D4 by $34.5 \%$ and $16.5 \%(p=0.08$ and 0.19 , respectively, two-tailed $t$-test), respectively, at $0.1 \AA \mathrm{ns}^{-1}$. To test whether these differences become statistically significant at higher sampling of starting conformations, we also ran 20 SMD simulations at higher pulling speeds $\left(2 \AA \mathrm{ns}^{-1}\right)$ for each nanobody. Simulations performed at $2 \AA \mathrm{ns}^{-1}$ revealed significantly lower unbinding work values from N501Y/
E484K/K417N RBD for both H11-H4 and H11-D4 (14.7\% and $17.3 \%, p=0.0008$ and 0.0022 , respectively, two-tailed $t$ test; SI Appendix Figure S6E and F). Changes in unbinding work values can be attributed to the loss of a salt bridge and a hydrogen bond due to the E484K mutation. In contrast to H11-H4 and H11-D4, the binding strength of Ty1 increased by $71.8 \%$ (Figure 4C and D) for $0.1 \AA \mathrm{ns}^{-1}$, nearly twice as strong as the binding strength of other nanobodies and ACE2 to this mutant ( $p=0.008$, two-tailed $t$ test). This may be due to the formation of an extensive network of hydrophobic interactions between the RBD loop (on which K484 is located) and Ty1 (SI Appendix Figure S7). These results suggest that Tyl can be more effective than H11-H4 and H11D4 in neutralizing the Beta variant of SARS-CoV-2.

To investigate whether H11-H4 and H11-D4 binding can abrogate ACE2 binding to the N501Y and N501Y/E484K/ $\mathrm{K} 417 \mathrm{~N}$ mutants, we performed three sets of cMD simulations for the ACE2-RBD ${ }_{\text {mutant }}$-nanobody trimeric complex. Although $\mathrm{H} 11-\mathrm{H} 4$ was able to unbind ACE2 from WT RBD in all six simulations, it was able to unbind ACE2 only in 1 out of $3 \mathrm{~N} 501 \mathrm{Y} / \mathrm{E} 484 \mathrm{~K} / \mathrm{K} 417 \mathrm{~N}$ RBD simulations and not able to unbind ACE2 in the 3 N501Y RBD simulations (SI Appendix Figures S8-S10). While H11-H4 unbinding from WT RBD was never observed, the presence of ACE2 on RBD resulted in dissociation of $\mathrm{H} 11-\mathrm{H} 4$ from N501Y in 1 out of 3 simulations and from N501Y/E484K/K417N in 2 out of 3 simulations (SI Appendix Figure S11). In comparison, H11-D4 was able to displace ACE2 from N501Y in only 1 out of 3 simulations and unable to displace ACE2 from the N501Y/E484K/K417N mutant. Collectively, these results suggest that $\mathrm{H} 11-\mathrm{H} 4$ and H11-D4 are less effective in preventing ACE2 binding to the Alpha and Beta variants compared to the WT SARS-CoV-2. 


\section{CONCLUSIONS}

We performed an extensive in silico analysis to explore how nanobodies H11-H4, H11-D4, and Ty1 disrupt S-ACE2 interactions and whether these nanobodies can also neutralize the SARS-CoV-2 variants Alpha and Beta. Pulling nanobodies away from WT or mutant RBDs at velocities comparable to those applied at AFM experiments enabled us to estimate the nanobody binding strengths and how binding strength is affected by the RBD mutations. Furthermore, by docking the nanobodies (H11-H4 and H11-D4) onto the ACE2-RBD complex or docking ACE2 onto the RBD-nanobody complexes, we provided a mechanistic explanation of how nanobodies abrogate ACE2 binding from WT and mutant RBD.

Our simulations showed that the RBD binding strengths of the nanobodies and ACE2 are comparable. The binding of $\mathrm{H} 11-\mathrm{H} 4$ to RBD was able to dislocate ACE2 from its RBD binding site. Dislocation of ACE2 was facilitated by the electrostatic tug-of-war between $\mathrm{H} 11-\mathrm{H} 4$ and ACE2 due to having identically charged residues in close vicinity when both proteins are bound to RBD side by side. H11-D4, on the other hand, was able to abrogate ACE2 binding to a lesser extent. Because the Ty1 binding site overlaps with the ACE2 binding site on RBD, we anticipate that it neutralizes ACE2 binding by steric exclusion. Together, our MD simulations suggest that H11-H4 and Ty1 abrogate ACE2 binding via different mechanisms, whereas H11-D4 is possibly the least effective inhibitor for WT RBD among the three nanobodies investigated in this study. This conclusion is in accord with the experimental studies, ${ }^{5,6}$ which reported the lowest $K_{d}$ value for Ty1 and the highest for H11-D4 for the three nanobodies we investigated.

H11-H4's effectiveness in abrogating ACE2 binding was diminished by N501Y and N501Y/E484K/K417N mutations of RBD. H11-D4 was much less effective in abrogating ACE2 binding to the triple RBD mutant within our simulation lengths. Furthermore, a large decrease in H11-H4 and H11-D4 binding strength to RBD was observed for N501Y/E484K/ $\mathrm{K} 417 \mathrm{~N}$ mutations of RBD. These results are consistent with recent studies that reported a minimal and moderate impact of antibodies on Alpha and Beta variants. ${ }^{40-45}$ Strikingly, Ty1 binds to RBD of the Beta variant two times stronger than ACE2. Based on the high binding strength of Ty1 to N501Y/ $\mathrm{E} 484 \mathrm{~K} / \mathrm{K} 417 \mathrm{~N} \mathrm{RBD}$ in comparison to ACE2, we predict that Ty1 will be able to neutralize Beta variants by sterically blocking ACE2 binding.

Our study showcases an efficient procedure through which the effectiveness of current neutralizing nanobodies can be tested against the SARS-CoV-2 variants with multiple mutations on the $S$ protein. Our simulations also provided engineering principles of next-generation nanobodies to counteract the binding weakening effect due to $S$ protein mutations. The delta variant, characterized by L452R and $\mathrm{T} 478 \mathrm{~K}$, has recently become the dominant variant in the US and several other countries. Because the L452R mutation is located on the H11-H4, H11-D4, and Tyl binding interface, our results indicate that this mutation may reduce the affinity of H11-H4, H11-D4, and Tyl binding. Future MD simulations will be required to investigate the effect of these mutations on nanobody binding.

Because complete glycan structures are invisible in structural studies, we used the visible parts of the glycans on the $S$ protein surface in our MD simulations. Although the precise length and composition of glycans remain unknown, a recent study by Amaro and co-workers modeled the most probable glycans on the $S$ protein. ${ }^{29}$ These modeled glycans do not overlap and are not in close proximity to the nanobody binding sites, indicating that missing glycan regions may not affect our conclusions (SI Appendix Figure S12). Because recent studies have highlighted the role of glycans in shielding the protein surface, $^{29}$ full-length glycans can be considered in future simulations of the $S$ protein.

\section{DATA AND SOFTWARE AVAILABILITY}

All data and software is available upon request to the corresponding author.

\section{ASSOCIATED CONTENT}

\section{(s) Supporting Information}

The Supporting Information is available free of charge at https://pubs.acs.org/doi/10.1021/acs.jcim.1c00695.

Structural and sequence information on nanobodies; Effect of H11-H4 and H11-D4 binding on interactions and interaction types between ACE2 and RBD; Rootmean-square deviation of the $\mathrm{RBD} \mathrm{C}_{\alpha}$ atoms upon nanobody binding in the presence of ACE2; Interactions between the N501Y mutant of RBD and the nanobodies; Average work required to unbind nanobodies or ACE2 under $2 \AA \mathrm{ns}^{-1}$ pulling speed; Changes of the interaction network between Tyl and RBD upon $\mathrm{N} 501 \mathrm{Y} / \mathrm{E} 484 \mathrm{~K} / \mathrm{K} 417 \mathrm{~N}$ mutation; Effect of $\mathrm{H} 11-\mathrm{H} 4$ and H11-D4 docking on interactions and interaction types between ACE2 and RBD variants; H11-H4 detaching from the ACE2-RBD complexes; Position of glycans of full-length $S$ protein and nanobodies; Performed MD simulations list; Steered and fixed atoms in SMD simulations (PDF)

Supplementary Movie S1 showing the electrostatic repulsion between ACE2 and $\mathrm{H} 11-\mathrm{H} 4$ upon $\mathrm{H} 11-\mathrm{H} 4$ docking and ACE2 discolation for WT and variants (MP4)

\section{AUTHOR INFORMATION}

\section{Corresponding Author}

Mert Gur - Department of Mechanical Engineering, Istanbul Technical University (ITU), 34437 Istanbul, Turkey; 이 orcid.org/0000-0003-0983-4397; Email: gurme@ itu.edu.tr

\section{Authors}

Mert Golcuk - Department of Mechanical Engineering, Istanbul Technical University (ITU), 34437 Istanbul, Turkey; (1) orcid.org/0000-0001-5476-8160

Aysima Hacisuleyman - Institute of Bioengineering, Swiss Federal Institute of Technology (EPFL), 1015 Lausanne, Switzerland; O orcid.org/0000-0003-2471-0296

Burak Erman - Chemical and Biological Engineering Department, Koc University, 34450 Istanbul, Turkey; (1) orcid.org/0000-0002-2496-6059

Ahmet Yildiz - Physics Department and Department of Molecular and Cell Biology, University of California, Berkeley, California 94720, United States

Complete contact information is available at: https://pubs.acs.org/10.1021/acs.jcim.1c00695 


\section{Author Contributions}

Mert Gur, A.Y., and B.E. initiated the project. Mert Gur supervised the project. Mert Golcuk, A.H., and Mert Gur performed MD simulations. Mert Golcuk, A.H., A.Y., and Mert Gur prepared the manuscript.

\section{Funding}

This work is supported by COVID-19 HPC Consortium (Grant number: TG-BIO200053) and the National Institute of Health (R35 GM136414; A.Y.).

\section{Notes}

The authors declare no competing financial interest.

\section{ACKNOWLEDGMENTS}

This work used resources services and support provided via the COVID-19 HPC Consortium (https://covid19-hpcconsortium.org/) and the Extreme Science and Engineering Discovery Environment (XSEDE), which is supported by National Science Foundation grant number ACI-1548562. A.Y. acknowledges to support of the National Institute of Health (1R35GM136414).

\section{ABBREVIATIONS}

$\mu \mathrm{s}$, microsecond; ACE2, angiotensin-converting enzyme 2; atm, standard atmosphere; $\mathrm{C}_{\alpha}$, alpha carbon; cMD, conventional molecular dynamics; COVID-19, coronavirus disease 2019; CR, contact region; fs, femtosecond; MD, molecular dynamics; NAMD, nanoscale molecular dynamics; ns, nanosecond; PD, peptidase domain; ps, picosecond; RBD, receptorbinding domain; RMSF, root-mean-square fluctuation; RNA, ribonucleic acid; S, spike; SARS-CoV, severe acute respiratory syndrome-coronavirus; SMD, steered molecular dynamics; VMD, visual molecular dynamics; WT, wild-type.

\section{REFERENCES}

(1) Bannas, P.; Hambach, J.; Koch-Nolte, F. Nanobodies and Nanobody-Based Human Heavy Chain Antibodies as Antitumor Therapeutics. Front. Immunol. 2017, 8, 1603.

(2) Gonzalez-Sapienza, G.; Rossotti, M. A.; Tabares-da Rosa, S. Single-Domain Antibodies as Versatile Affinity Reagents for Analytical and Diagnostic Applications. Front. Immunol. 2017, 8, 977.

(3) Muyldermans, S. A Guide to: Generation and Design of Nanobodies. FEBS J. 2021, 288, 2084-2102.

(4) Noël, F.; Malpertuy, A.; de Brevern, A. G. Global Analysis of VHHs Framework Regions with a Structural Alphabet. Biochimie 2016, 131, 11-19.

(5) Huo, J.; Le Bas, A.; Ruza, R. R.; Duyvesteyn, H. M.; Mikolajek, H.; Malinauskas, T.; Tan, T. K.; Rijal, P.; Dumoux, M.; Ward, P. N. Neutralizing nanobodies bind SARS-CoV-2 spike RBD and block interaction with ACE2. Nat. Struct. Mol. Biol. 2020, 27, 846-854.

(6) Hanke, L.; Vidakovics Perez, L.; Sheward, D. J.; Das, H.; Schulte, T.; Moliner-Morro, A.; Corcoran, M.; Achour, A.; Karlsson Hedestam, G. B.; Hallberg, B. M.; Murrell, B.; McInerney, G. M. An alpaca nanobody neutralizes SARS-CoV-2 by blocking receptor interaction. Nat. Commun. 2020, 11, 1-9.

(7) Xiang, Y.; Nambulli, S.; Xiao, Z.; Liu, H.; Sang, Z.; Duprex, W. P.; Schneidman-Duhovny, D.; Zhang, C.; Shi, Y. Versatile and Multivalent Nanobodies Efficiently Neutralize SARS-CoV-2. Science 2020, 370, 1479-1484.

(8) Schoof, M.; Faust, B.; Saunders, R. A.; Sangwan, S.; Rezelj, V.; Hoppe, N.; Boone, M.; Billesbølle, C. B.; Puchades, C.; Azumaya, C. M.; et al. An Ultrapotent Synthetic Nanobody Neutralizes SARSCoV-2 by Stabilizing Inactive Spike. Science 2020, 370, 1473-1479.

(9) Custódio, T. F.; Das, H.; Sheward, D. J.; Hanke, L.; Pazicky, S.; Pieprzyk, J.; Sorgenfrei, M.; Schroer, M. A.; Gruzinov, A. Y.; Jeffries, C. M.; et al. Selection, biophysical and structural analysis of synthetic nanobodies that effectively neutralize SARS-CoV-2. Nat. Commun. 2020, 11, 1-11.

(10) Belouzard, S.; Millet, J. K.; Licitra, B. N.; Whittaker, G. R. Mechanisms of coronavirus cell entry mediated by the viral spike protein. Viruses 2012, 4, 1011-1033.

(11) Shang, J.; Wan, Y.; Luo, C.; Ye, G.; Geng, Q.; Auerbach, A.; Li, F. Cell entry mechanisms of SARS-CoV-2. Proc. Natl. Acad. Sci. U. S. A. 2020, 117, 11727-11734.

(12) Faria, N. R.; Claro, I. M.; Candido, D.; Moyses Franco, L. A.; Andrade, P. S.; Coletti, T. M.; Silva, C. A. M.; Sales, F. C.; Manuli, E. R., Aguiar, R. S., Gaburo, N.; Camilo, C. da C.; Fraiji, N. A.; Esashika Crispim, M. A.; Carvalho, M. S. S.; Rambaut, A.; Loman, N.; Pybus, O. G.; Sabino, E. C.; on behalf of CADDE Genomic Network. Genomic Characterisation of an Emergent SARS-CoV-2 Lineage in Manaus: Preliminary Findings. https://virological.org/t/genomiccharacterisation-of-an-emergent-sars-cov-2-lineage-in-manauspreliminary-findings/586 (accessed 12/05/2021).

(13) Lan, J.; Ge, J.; Yu, J.; Shan, S.; Zhou, H.; Fan, S.; Zhang, Q.; Shi, X.; Wang, Q.; Zhang, L.; Wang, X. Structure of the SARS-CoV-2 Spike Receptor-Binding Domain Bound to the ACE2 Receptor. Nature 2020, 581, 215-220.

(14) Taka, E.; Yilmaz, S. Z.; Golcuk, M.; Kilinc, C.; Aktas, U.; Yildiz, A.; Gur, M. Critical Interactions Between the SARS-CoV-2 Spike Glycoprotein and the Human ACE2 Receptor. J. Phys. Chem. B 2021, $125,5537-5548$.

(15) Isralewitz, B.; Gao, M.; Schulten, K. Steered Molecular Dynamics and Mechanical Functions of Proteins. Curr. Opin. Struct. Biol. 2001, 11, 224-230.

(16) Humphrey, W.; Dalke, A.; Schulten, K. VMD: visual molecular dynamics. J. Mol. Graphics 1996, 14, 33-38.

(17) Phillips, J. C.; Hardy, D. J.; Maia, J. D.; Stone, J. E.; Ribeiro, J. V.; Bernardi, R. C.; Buch, R.; Fiorin, G.; Hénin, J.; Jiang, W.; et al. Scalable Molecular Dynamics on CPU and GPU Architectures with NAMD. J. Chem. Phys. 2020, 153, No. 044130.

(18) Best, R. B.; Zhu, X.; Shim, J.; Lopes, P. E.; Mittal, J.; Feig, M.; MacKerell, A. D., Jr Optimization of the Additive CHARMM AllAtom Protein Force Field Targeting Improved Sampling of The Backbone $\phi, \psi$ and Side-Chain $\chi 1$ and $\chi 2$ Dihedral Angles. J. Chem. Theory Comput. 2012, 8, 3257-3273.

(19) Pullara, F.; Wenzhi, M.; Gür, M. Why Protein Conformers in Molecular Dynamics Simulations Differ from Their Crystal Structures: A Thermodynamic Insight. Turk. J. Chem. 2019, 43, 394-403.

(20) Beckstein, O.; Denning, E. J.; Perilla, J. R.; Woolf, T. B. Zipping and unzipping of adenylate kinase: atomistic insights into the ensemble of open $\leftrightarrow$ closed transitions. J. Mol. Biol. 2009, 394, $160-176$.

(21) Durrant, J. D.; McCammon, J. A. HBonanza: a computer algorithm for molecular-dynamics-trajectory hydrogen-bond analysis. J. Mol. Graphics Modell. 2011, 31, 5-9.

(22) Stock, P.; Utzig, T.; Valtiner, M. Direct and Quantitative AFM Measurements of the Concentration and Temperature Dependence of the Hydrophobic Force Law at Nanoscopic Contacts. J. Colloid Interface Sci. 2015, 446, 244-251.

(23) Manavalan, P.; Ponnuswamy, P. A Study of the Preferred Environment of Amino Acid Residues in Globular Proteins. Arch. Biochem. Biophys. 1977, 184, 476-487.

(24) Stavrakoudis, A.; Tsoulos, I. G.; Shenkarev, Z. O.; Ovchinnikova, T. V. Molecular Dynamics Simulation of Antimicrobial Peptide Arenicin-2: b-Hairpin Stabilization by Noncovalent Interactions. Biopolymers 2009, 92, 143-155.

(25) Phillips, J. C.; Braun, R.; Wang, W.; Gumbart, J.; Tajkhorshid, E.; Villa, E.; Chipot, C.; Skeel, R. D.; Kale, L.; Schulten, K. Scalable Molecular Dynamics with NAMD. J. Comput. Chem. 2005, 26, 17811802.

(26) Park, S.; Khalili-Araghi, F.; Tajkhorshid, E.; Schulten, K. Free energy calculation from steered molecular dynamics simulations using Jarzynski's equality. J. Chem. Phys. 2003, 119, 3559-3566. 
(27) Wang, Y.; Liu, M.; Gao, J. Enhanced Receptor Binding of SARS-CoV-2 Through Networks of Hydrogen-Bonding and Hydrophobic Interactions. Proc. Natl. Acad. Sci. U. S. A. 2020, 117, 1396713974.

(28) Ahmed, M. C.; Papaleo, E.; Lindorff-Larsen, K. How well do force fields capture the strength of salt bridges in proteins? PeerJ 2018, 6, No. e4967.

(29) Casalino, L.; Gaieb, Z.; Goldsmith, J. A.; Hjorth, C. K.; Dommer, A. C.; Harbison, A. M.; Fogarty, C. A.; Barros, E. P.; Taylor, B. C.; McLellan, J. S.; Fadda, E.; Amaro, R. E. Beyond Shielding: The Roles of Glycans in the SARS-CoV-2 Spike Protein. ACS Cent. Sci. 2020, 6, 1722-1734.

(30) Huang, S.; Zhang, D.; Mei, H.; Kevin, M.; Qu, S.; Pan, X.; Lu, L. SMD-Based Interaction-Energy Fingerprints Can Predict Accurately the Dissociation Rate Constants of HIV-1 Protease Inhibitors. J. Chem. Inf. Model. 2019, 59, 159-169.

(31) Moore, D. S.; Dalton, J. P.; Tikhonova, I. G. Steered Molecular Dynamic Simulations Reveal Critical Residues for (Un) Binding of Substrates, Inhibitors and a Product of the Malarial PFM1AAP. Biophys. J. 2017, 112, 354a.

(32) Kosztin, D.; Izrailev, S.; Schulten, K. Unbinding of Retinoic Acid from Its Receptor Studied by Steered Molecular Dynamics. Biophys. J. 1999, 76, 188-197.

(33) Azadi, S.; Tafazzoli-Shadpour, M.; Omidvar, R. Steered Molecular Dynamics Simulation Study of Quantified Effects of Point Mutation Induced by Breast Cancer on Mechanical Behavior of E-Cadherin. Mol. Biol. 2018, 52, 723-731.

(34) Hu, X.; Hu, S.; Wang, J.; Dong, Y.; Zhang, L.; Dong, Y. Steered Molecular Dynamics for Studying Ligand Unbinding of Ecdysone Receptor. J. Biomol. Struct. Dyn. 2018, 36, 3819-3828.

(35) Cao, W.; Dong, C.; Kim, S.; Hou, D.; Tai, W.; Du, L.; Im, W.; Zhang, X. F. Biomechanical characterization of SARS-CoV-2 spike $\mathrm{RBD}$ and human ACE2 protein-protein interaction. Biophys. J. 2021, 120, 1011-1019.

(36) Gur, M.; Taka, E.; Yilmaz, S. Z.; Kilinc, C.; Aktas, U.; Golcuk, M. Conformational Transition of SARS-CoV-2 Spike Glycoprotein Between Its Closed and Open States. J. Chem. Phys. 2020, 153, No. 075101.

(37) Eskici, G.; Gur, M. Computational design of new peptide inhibitors for amyloid beta (A $\beta)$ aggregation in Alzheimer's disease: application of a novel methodology. PLoS One 2013, 8, No. e66178.

(38) Nguyen, H. L.; Lan, P. D.; Thai, N. Q.; Nissley, D. A.; O’Brien, E. P.; Li, M. S. Does SARS-CoV-2 Bind to Human ACE2More Strongly Than Does SARS-CoV? J. Phys. Chem. B 2020, 124, 73367347.

(39) Rico, F.; Gonzalez, L.; Casuso, I.; Puig-Vidal, M.; Scheuring, S. High-Speed Force Spectroscopy Unfolds Titin at the Velocity of Molecular Dynamics Simulations. Science 2013, 342, 741-743.

(40) Wang, P.; Nair, M. S.; Liu, L.; Iketani, S.; Luo, Y.; Guo, Y.; Wang, M.; Yu, J.; Zhang, B.; Kwong, P. D.; et al. Antibody Resistance of SARS-CoV-2 Variants B. 1.351 and B. 1.1. 7. Nature 2021, 593, 130-135.

(41) Shen, X.; Tang, H.; McDanal, C.; Wagh, K.; Fischer, W.; Theiler, J.; Yoon, H.; Li, D.; Haynes, B.; et al. SARS-CoV-2 Variant B. 1.1. 7 is Susceptible to Neutralizing Antibodies Elicited by Ancestral Spike Vaccines. Cell Host Microbe 2021, 29, 529-539.

(42) Edara, V. V.; Floyd, K.; Lai, L.; Gardner, M.; Hudson, W.; Piantadosi, A.; Waggoner, J.; Babiker, A.; Ahmed, R.; Xie, X. Infection and mRNA-1273 vaccine antibodies neutralize SARS-CoV-2 UK variant. medRxiv 2021, DOI: 10.1101/2021.02.02.21250799.

(43) Wu, K.; Werner, A. P.; Moliva, J. I.; Koch, M.; Choi, A.; Stewart-Jones, G. B.; Bennett, H.; Boyoglu-Barnum, S.; Shi, W.; Graham, B. S. mRNA-1273 vaccine induces neutralizing antibodies against spike mutants from global SARS-CoV-2 variants. BioRxiv 2021, DOI: $10.1101 / 2021.01 .25 .427948$.

(44) Emary, K. R.; Golubchik, T.; Aley, P. K.; Ariani, C. V.; Angus, B.; Bibi, S.; Blane, B.; Bonsall, D.; Cicconi, P.; Charlton, S.; et al. Efficacy of ChAdOx1 nCoV-19 (AZD1222) Vaccine Against SARSCoV-2 Variant of Concern 202012/01 (B. 1.1. 7): An Exploratory
Analysis of a Randomised Controlled Trial. Lancet 2021, 397, 13511362.

(45) Collier, D. A.; De Marco, A.; Ferreira, I. A.; Meng, B.; Datir, R. P.; Walls, A. C.; Kemp, S. A.; Bassi, J.; Pinto, D.; Silacci-Fregni, C.; et al. Sensitivity of SARS-CoV-2 B. 1.1. 7 to mRNA Vaccine-Elicited Antibodies. Nature 2021, 593, 136-141. 